\title{
Self-management interventions for chronic kidney disease: a systematic review and meta-analysis
}

Suyuan Peng ${ }^{1,2}$, Jiawei He ${ }^{3}$, Jiasheng Huang ${ }^{1}$, Longwei Lun ${ }^{4}$, Jiahao Zeng ${ }^{1}$, Shan Zeng ${ }^{1}$, La Zhang ${ }^{6,7}$, Xusheng $\mathrm{Liu}^{4}$ and Yifan $\mathrm{Wu}^{5^{*}}$ (D)

\begin{abstract}
Background: Self-management intervention aims to facilitate an individual's ability to make lifestyle changes. The effectiveness of this intervention in non-dialysis patients with chronic kidney disease (CKD) is limited. In this study, we applied a systematic review and meta-analysis to investigate whether self-management intervention improves renoprotection for non-dialysis chronic kidney disease.

Methods: We conducted a comprehensive search for randomized controlled trials addressing our objective. We searched for studies up to May 12, 2018. Two reviewers independently evaluated study quality and extracted characteristics and outcomes among patients with CKD within the intervention phase for each trial. Meta-regression and subgroup analyses were conducted to explore heterogeneity.

Results: We identified 19 studies with a total of 2540 CKD patients and a mean follow-up of 13.44 months. Compared with usual care, self-management intervention did not show a significant difference for risk of all-cause mortality (5 studies, 1662 participants; RR 1.13; 95\% Cl 0.68 to 1.86; $I^{2}=0 \%$ ), risk of dialysis (5 studies, 1565 participants; RR $1.35 ; 95 \% \mathrm{Cl} 0.84$ to $2.19 ; \mathrm{I}^{2}=0 \%$ ), or change in eGFR (8 studies, 1315 participants; SMD $-0.01 ; 95 \% \mathrm{Cl}-0.23$ to 0.21 ; $\left.\mathrm{I}^{2}=64 \%\right)$. Moreover, self-management interventions were associated with a lower $24 \mathrm{~h}$ urinary protein excretion (4 studies, 905 participants; MD $-0.12 \mathrm{~g} / 24 \mathrm{~h} ; 95 \% \mathrm{Cl}-0.21$ to $-0.02 ; \mathrm{l}^{2}=3 \%$ ), a lower blood pressure level (SBP: 7 studies, 1201 participants; MD $-5.68 \mathrm{mmHg} ; 95 \% \mathrm{Cl}-9.68$ to $-1.67 ; \mathrm{I}^{2}=60 \%$; DBP: 7 studies, 1201 participants; $\mathrm{MD}-2.64 \mathrm{mmHg}, 95 \% \mathrm{Cl}-3.78$ to $-1.50 ;\left.\right|^{2}=0 \%$ ), a lower C-reactive Protein (CRP) level (3 studies, 123 participants; $\mathrm{SMD}-2.8 ; 95 \% \mathrm{Cl}-2.90$ to $-2.70 ; \mathrm{I}^{2}=0 \%$ ) and a longer distance on the 6-min walk (3 studies, 277 participants; SMD $0.70 ; 95 \% \mathrm{Cl} 0.45$ to $0.94 ; 1^{2}=0 \%$ ) when compared with the control group.
\end{abstract}

Conclusions: We observed that self-management intervention was beneficial for urine protein decline, blood pressure level, exercise capacity and CRP level, compared with the standard treatment, during a follow-up of 13.44 months in patients with CKD non-dialysis. However, it did not provide additional benefits for renal outcomes and all-cause mortality.

Keywords: Chronic kidney disease, Self-management, Chronic disease management

\footnotetext{
* Correspondence: wuyifan007@gzucm.edu.cn

${ }^{5}$ Chronic Disease Management Department, Guangdong Provincial Hospital

of Chinese Medicine, The Second Affiliated Hospital of Guangzhou University

of Chinese Medicine, No. 111, Dade Rd, Yuexiu District, Guangzhou,

Guangdong Province, China

Full list of author information is available at the end of the article
}

(c) The Author(s). 2019 Open Access This article is distributed under the terms of the Creative Commons Attribution 4.0 International License (http://creativecommons.org/licenses/by/4.0/), which permits unrestricted use, distribution, and reproduction in any medium, provided you give appropriate credit to the original author(s) and the source, provide a link to the Creative Commons license, and indicate if changes were made. The Creative Commons Public Domain Dedication waiver (http://creativecommons.org/publicdomain/zero/1.0/) applies to the data made available in this article, unless otherwise stated. 


\section{Background}

Chronic kidney disease (CKD) is a progressive disease that leads to End-Stage Renal Disease (ESRD: maintenance dialysis or kidney transplantation), cardiovascular morbidity and mortality [1]. Approximately 440,000 patients begin dialysis each year worldwide, and annual costs of dialysis and kidney transplants range between US \$35,000 and 100,000. This can strain healthcare budgets [2]. Clinical decision making for CKD is challenging [3] due to the heterogeneity of kidney diseases, variability in rates of disease progression, and the competing risk of cardiovascular mortality, the most common cause of death worldwide [4]. Furthermore, CKD is not included in the list of priorities for most non-communicable diseases (NCD), and few countries have clear policies or public programs to prevent and control CKD [5].

CKD management includes slowing the progression to ESRD and decreasing the risk of cardiovascular complications through management of kidney function and CKD progression risk factors such as hypertension and diabetes [6]. In addition to medication, managing risk factors is important clinically because it can prevent, or at least minimize, the likelihood of further renal injury. Long-term CKD management requires a high level of patient involvement, both in decision-making and in the implementation of care. There is growing recognition that patients want to be involved as equal partners in their care. The goal of self-management is to identify strategies that can be used to help patients manage their condition(s) while leading active and productive lives. This includes goal setting, problem solving, symptom management, and shared decision-making, and these strategies are applicable for a diverse population [7]. For patients with CKD, this encompasses a spectrum of behaviors ranging from adherence to medication, exercise, and diet recommendations (self-management maintenance) to recognition of early warning signs, and self-adjustment of home-care regimens.

Despite an established tradition of patient self-management of ESRD, and self-management being a well-established treatment strategy for other chronic conditions such as diabetes [8, 9] and hypertension [10], evidence to support its use for CKD non-dialysis is limited. To lighten the economic burden of ESRD, strategies must be implemented to prevent the progression from early-stage CKD. In this systematic review and meta-analysis, we synthesized results from RCTs to evaluate the effects of self-management intervention on major renal outcomes and mortality in non-dialysis adults with CKD. We also assessed effect modification by proteinuria and blood pressure.

\section{Methods}

We performed a systematic review according to a specified protocol (PROSPERO number: CRD42017059870
[11]). The review reported in accordance with the Preferred Reporting Items for Systematic Reviews and Meta-Analyses (PRISMA) [12] statement recommendations.

\section{Literature search}

Electronic databases were searched using a strategy combining selected $\mathrm{MeSH}$ terms with keywords related to CKD and self-management intervention. We used English and Chinese language restriction [11].

Relevant studies were identified by searching the following electronic databases from inception to 12th of May 2018: PubMed, MEDLINE, EMBASE, CINAHL, the Cochrane Library database, the Chinese Biomedicine Database (CBM), Chinese National Knowledge Infrastructure (CNKI), and Wanfang Database. Reference lists from relevant review articles and reviews were also searched.

Studies were first screened according to title and abstract, and the full texts of any study considered relevant according to the selection criteria were assessed for eligibility by 2 independent reviewers (JS. $\mathrm{H}$ and JW. H). Disagreements between the reviewers concerning decisions to include or exclude studies were resolved by consensus, and if necessary, consultation with a third reviewer (YF. W).

\section{Selection criteria}

We included randomized controlled trials of self-management intervention compared with usual care for adults (age 18 and above) who had been clinically diagnosed with chronic kidney disease. CKD was defined as a glomerular filtration rate $($ GFR $)<60 \mathrm{~mL} / \mathrm{min} / 1.73 \mathrm{~m}^{2}$ or markers of kidney damage, or both, of at least 3 months duration [13]. We included interventions employing self-efficacy training, empowerment, cognitive behavioral therapy, or educational programs focusing on self-management, delivered either face-to-face or through telehealth sessions. Eligible studies had to have been published as full-length articles in peer-reviewed journals. Patients currently receiving renal replacement therapy [RRT] (dialysis or kidney transplantation) were excluded.

\section{Outcomes}

Comparing self-management intervention with the standard CKD treatment during the follow-up period, the primary outcomes of this systematic review included all-cause mortality, number of patients progressing to ESRD, change in GFR, change in proteinuria excretion, and adverse events. Secondary outcomes included health literacy (diet modifications, exercise capacity) and other indexes of CKD risk factors, including glycaemia, blood pressure, blood lipid concentration and C-reactive protein (CRP) level. 
Data extraction and quality assessment

Data extraction included details on the study characteristics (country, study design, sample size and study duration), population characteristics (age, sex, CKD stage), intervention (intervention format, length and delivery) and theoretical frameworks. The comparators and outcomes assessed were extracted, and then tabulated.

We used the recommended Cochrane risk of bias assessment tool [14], and the following items were assessed: random sequence generation, allocation concealment, blinding of participants and personnel, blinding of outcome assessment, incomplete outcome data and selective outcome reporting.

\section{Data synthesis and analysis}

Because of the between-study variance, we used a random-effects model for all analyses [15]. Effects were reported as the relative risk (RR) and 95\% confidence intervals (CI) for dichotomous outcomes, and mean difference (MD) or standard mean difference (SMD) and 95\% CI for continuous outcomes. The SMD was used when all studies had assess the same outcome, but had measured it differently (e.g. GFR was calculated by CKD-EPI equation or CKD-MDRD equation).

We examined the influence of various characteristics on the study-specific effect estimates by stratifying the analysis by self-management type: a) lifestyle modifications; b) medical-behavior modifications; c) multi-factorial modifications.

Statistical heterogeneity across studies was detected with the Cochrane $Q$ statistic and an $I^{2}$ test [14]. In cases with substantial heterogeneity, subgroup analysis and meta-regression were conducted to explore potential sources of variation. Subgroup analysis was conducted based on intervention format, treatment duration, and diabetic kidney disease populations.

Funnel plot is a useful tool to visually assess the potential for publication bias. If publication bias had been present, then smaller (less precise) studies that had failed to show statistical significance would have been less likely to have been published. This is reflected as asymmetry in the funnel plot. Publication bias was also examined by visual inspection of funnel plots for asymmetry and either Egger's linear regression test for dichotomous data [16, 17]' or Harbord's test [18] for continuous data. A $p$-value less than 0.05 was considered statistically significant. To assess the robustness of our meta-analyses, we conducted a trim and fill analysis. The trim and fill method is used to identify, and correct for, funnel plot asymmetry arising from publication bias.

Data were analyzed using RevMan 5.3.3 and STATA 14.0.

\section{Results}

Search yield

Computerized and manual searches resulted in 1737 unique citations, 1280 of which were excluded after reviewing their titles and abstracts. In total, 252 potentially eligible articles were retrieved for full-text review, and 233 articles were excluded. Both reviewers (HS. H. and JW. H.) agreed to include 19 publications in the present study. See Fig. 1 for details on the review process.

\section{Study and participant characteristics}

Nineteen studies, with a total of 2540 CKD patients, were deemed eligible. The clinical and methodological characteristics of each study are summarized in Table 1. The follow-up duration across studies ranged from 3 months to 60 months, and mean duration was 13.44 months. All study participants had CKD, and 10 studies were designed for participants with CKD and concomitant diabetes or hypertension. Studies took place in Europe (2 in the Netherlands, 2 in the UK), North America (3 in the US, 2 in Canada), Asia (2 in Taiwan, 1 in Hong Kong, 1 in Japan), Oceania (4 in Australia, 1 in New Zealand) and Africa (1 in Algeria).

\section{Intervention features}

Trials in this review comprised various kinds of self-management. We grouped trials into similar interventions (lifestyle modifications; medical-behavior modifications and multi-factorial modifications). Lifestyle interventions were the most common, followed by medical related practice and multi-factorial interventions. Among the lifestyle intervention trials, 9 studies included interventions related to lifestyle modification, targeting nutrition management, weight management or physical exercise. Four studies included in interventions were related to medical-behavior modification, targeting medicine adherence, disease cognition and complication control; and 6 were related to multi-factorial modifications (combined lifestyle and medical behaviors). The programs were delivered either face-to-face in an individual or group format, or via telehealth sessions (i.e. telephone, Digital Versatile Disc). They were conducted by a range of professionals, including nurses, dieticians/ nutritionists, certified exercise physiologists (CEP) and physicians (Table 1).

Six different self-management intervention theoretical frameworks were included in these articles: the Coventry, Aberdeen, and London-Refined (CALO-RE) taxonomy; components of the chronic care (CCM) model; the Trans-Theoretical Model (TTM), the Stanford Patient Education, the SMS program, and the Health Belief Model (HBM). When there was insufficient information, 


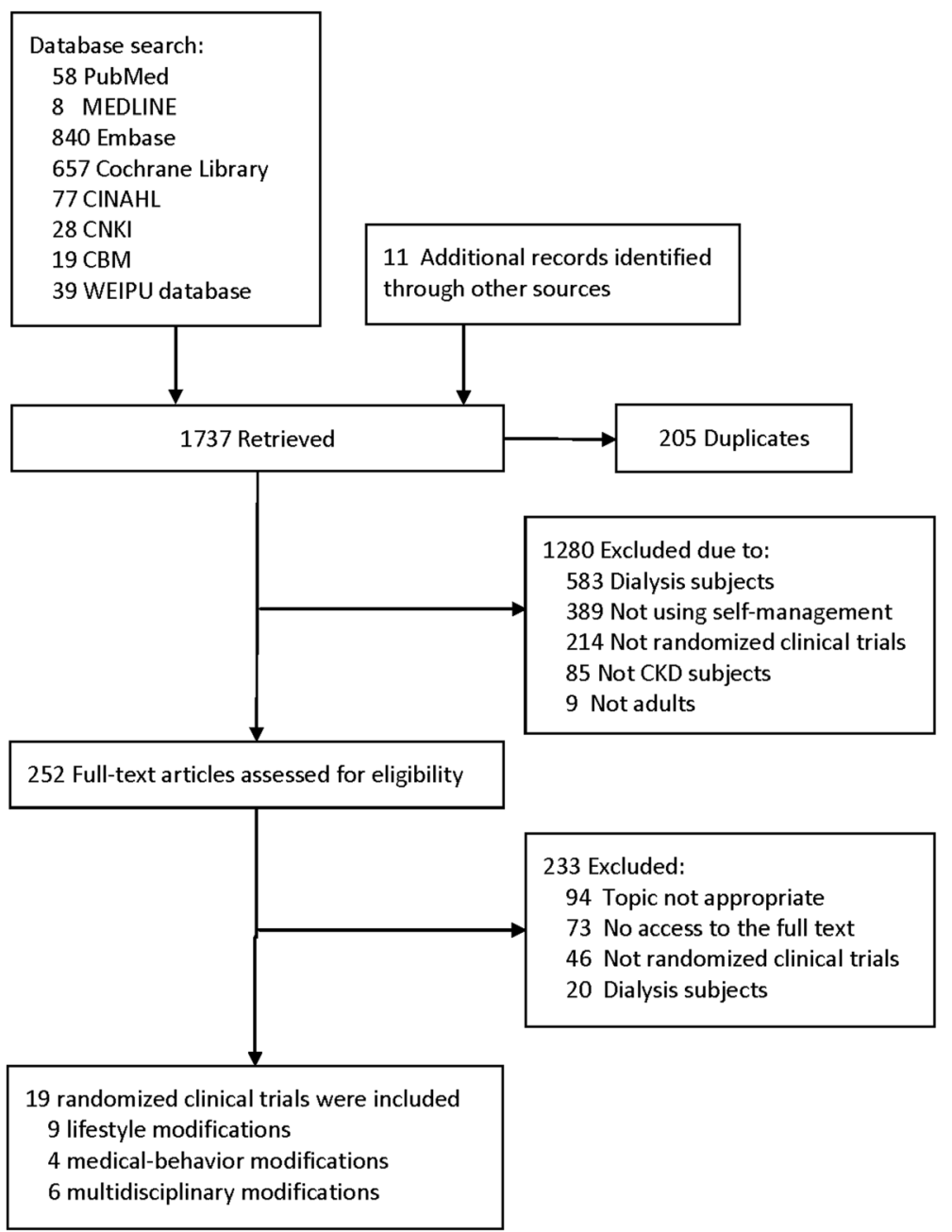

Fig. 1 Flow diagram of the stages of article inclusion for this systematic review

we attempted to contact the authors, but the response rate was poor.

\section{Quality assessment}

The risk of bias of included studies is summarized in Additional file 1: Figure S1 and S2. The main cause of potential bias was inadequate allocation concealment. Due to insufficient information, judgement could not be made in most of the studies regarding either allocation concealment or selection reporting.

\section{Effects of self-management intervention on kidney disease progression}

Figures 2, 3, 4 and 5 shows the pooled estimates for the primary outcomes. Compared with the standard treatment strategy, self-management intervention showed no significant difference in risk of all-cause mortality (5 studies, 1662 participants; RR 1.13; 95\% CI 0.68 to 1.86 ,
$\mathrm{I}^{2}=0 \%$ ) (Fig. 2), risk of dialysis (5 studies, 1565 participants; RR 1.35 ; $95 \%$ CI 0.84 to $2.19, \mathrm{I}^{2}=0 \%$ ) (Fig. 3 ), or change in GFR (7 studies, 1315 participants; SMD -0.01; $95 \%$ CI -0.23 to $0.21, \mathrm{I}^{2}=64 \%$ ) (Fig. 4). Moreover, self-management interventions were associated with a lower $24 \mathrm{~h}$ urinary protein excretion than that of the usual care (4 studies, 905 participants; MD - $0.12 \mathrm{~g} / 24 \mathrm{~h}$; 95\% CI -0.21 to $-0.02, \mathrm{I}^{2}=3 \%$ ) (Fig. 5).

The funnel plots, Egger's regression asymmetry test, and Harbord's regression asymmetry test each indicated no significant publication bias for any outcome. There was also no statistical heterogeneity for any of the outcomes (Additional file 1: Figure S3).

We also conducted a trim and fill analysis to determine the robustness of our meta-analysis. There was no effect of replacing missing studies, and the results showed that these estimates were robust and changed little (Additional file 1: Table S1). 


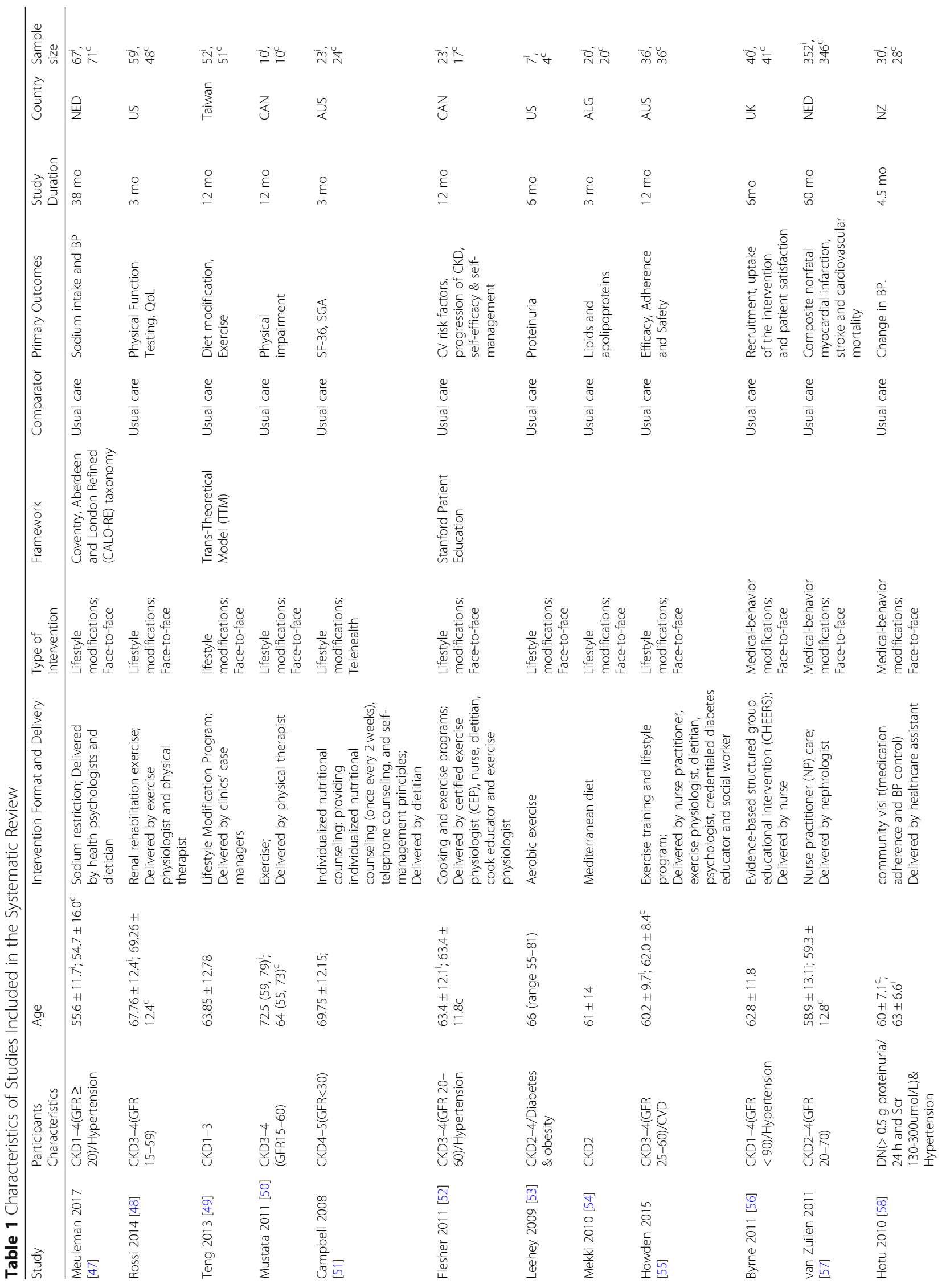




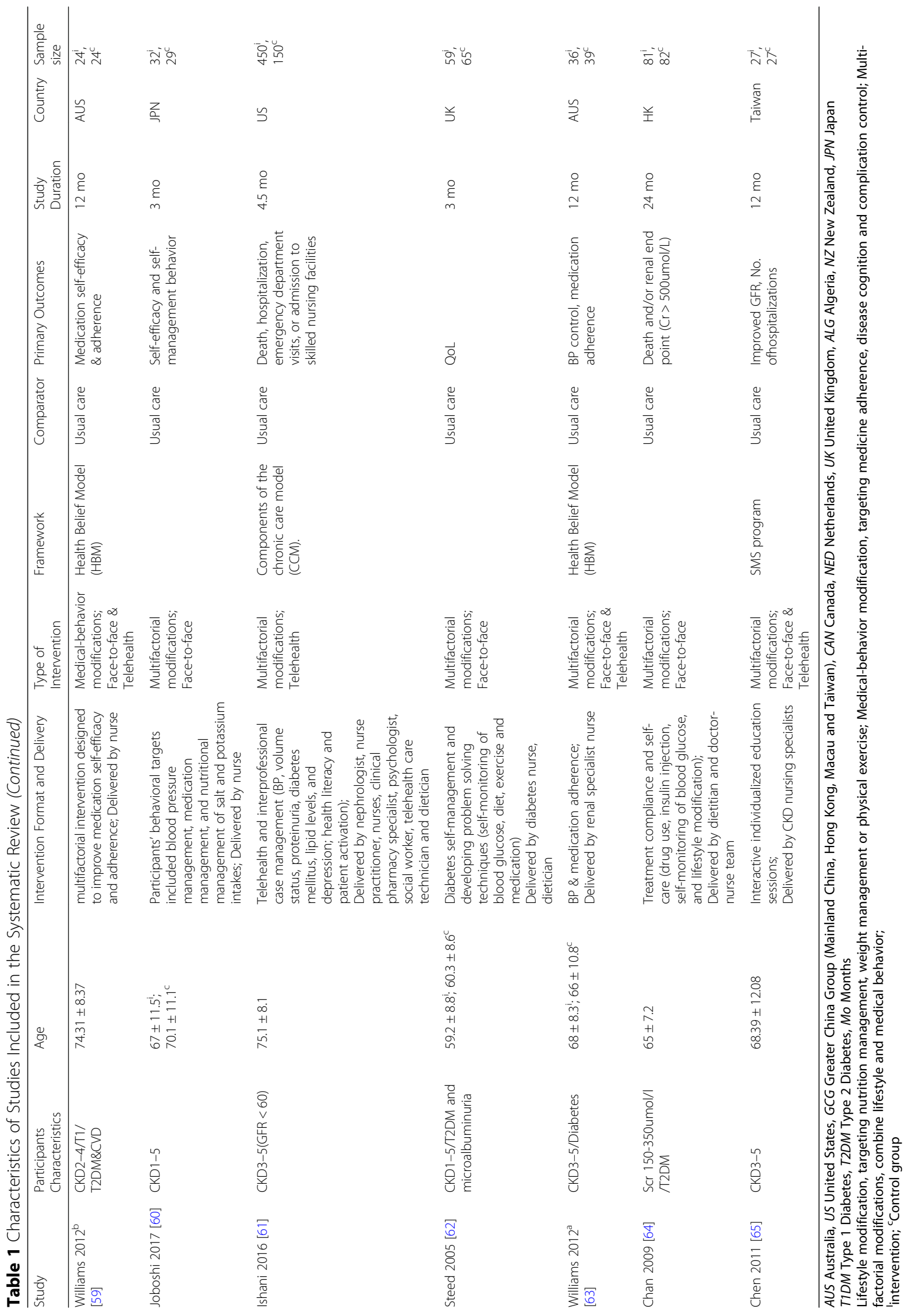




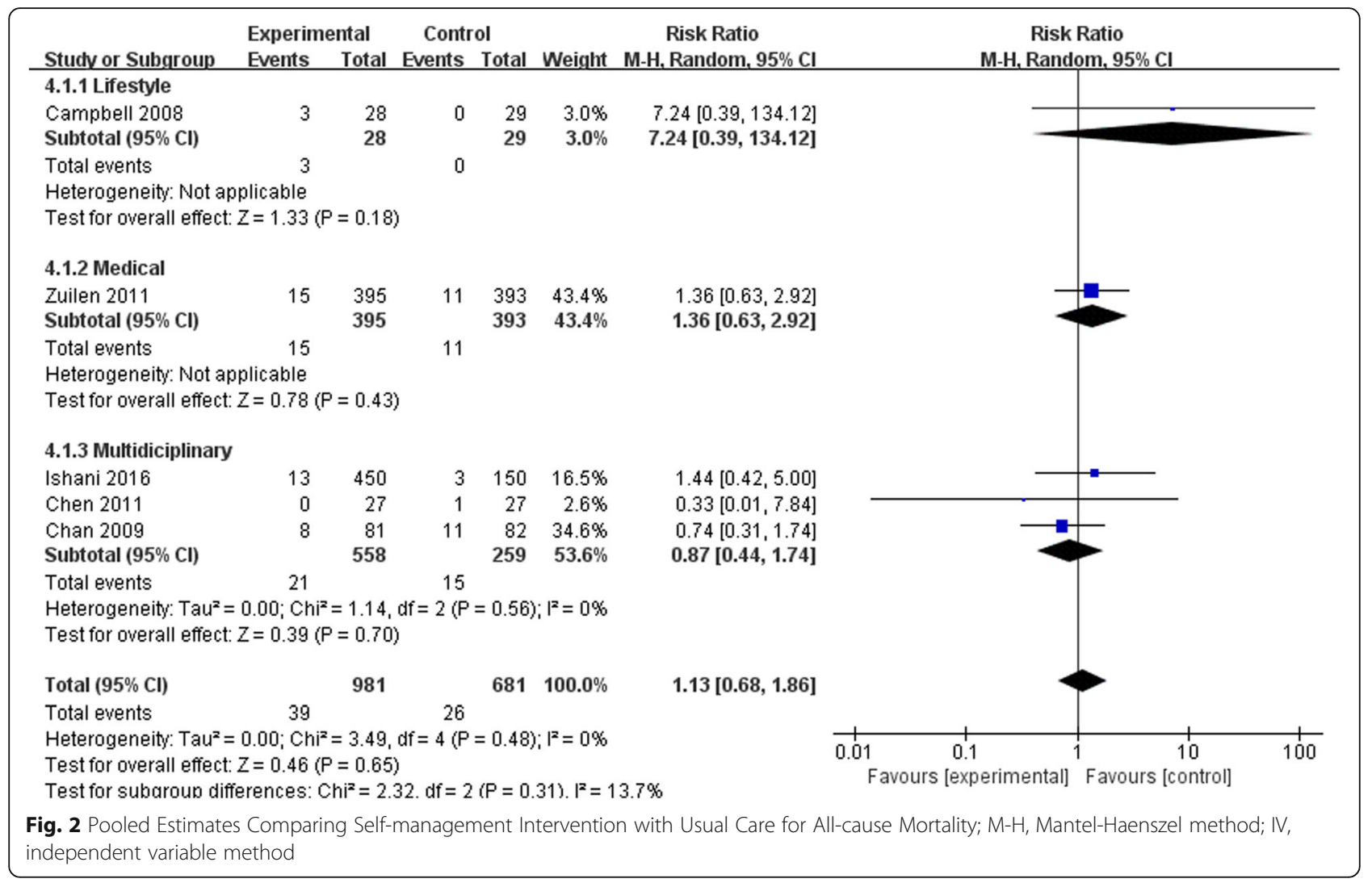

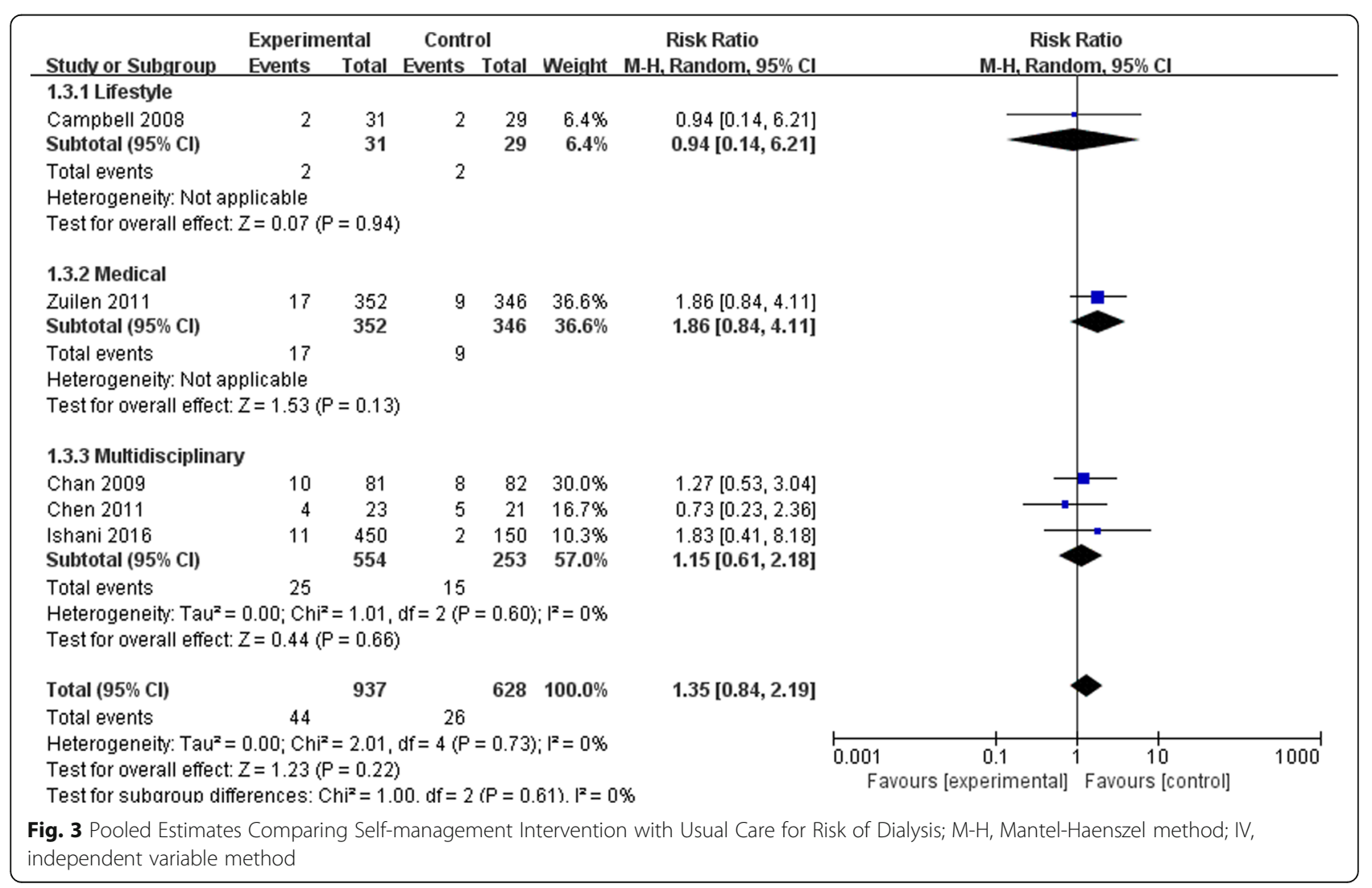




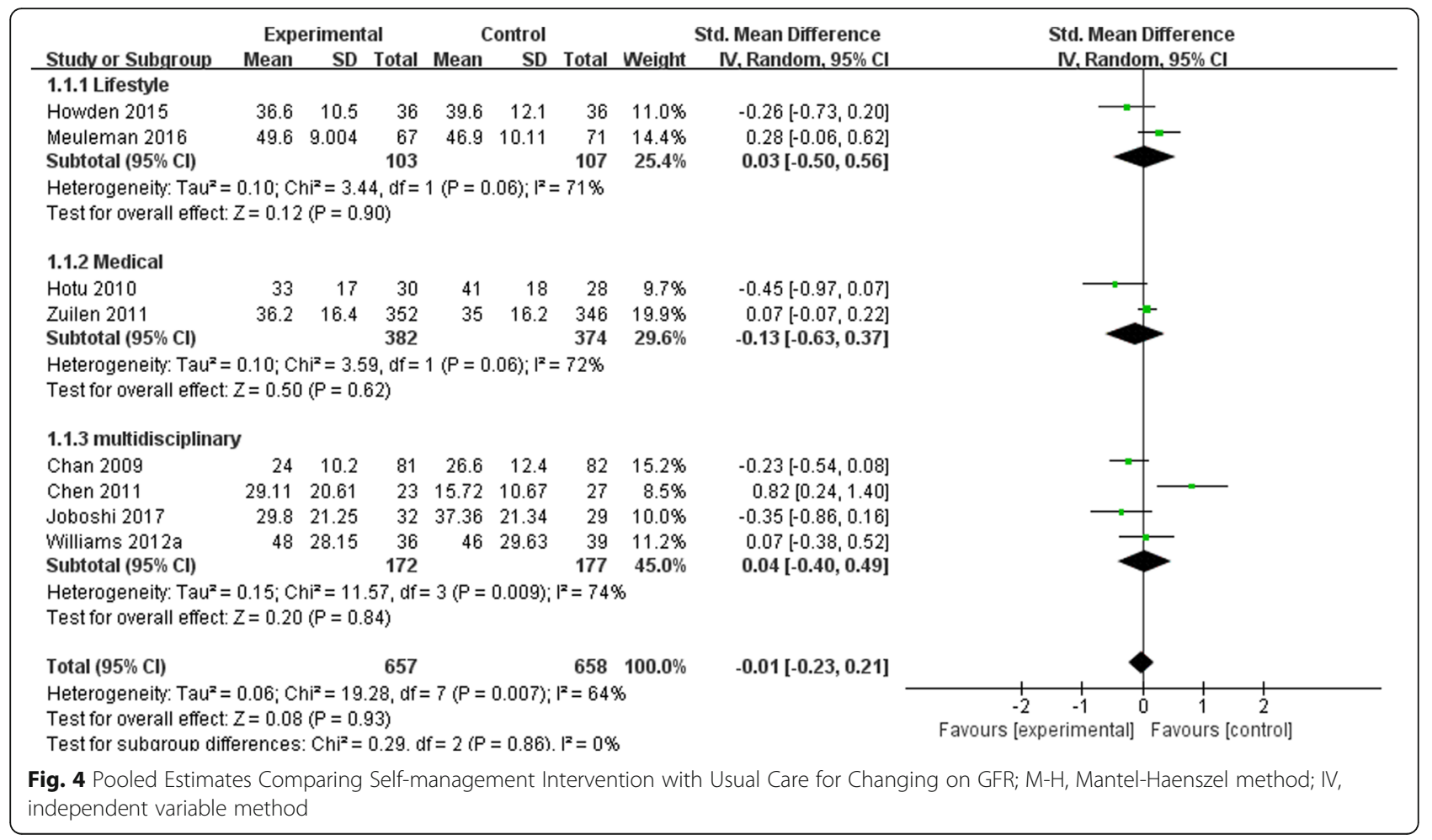

\section{Effects of self-management on risk factors}

For the surrogate outcomes, meta-analysis showed that self-management interventions were associated with a lower blood pressure level (SBP: 7 studies, 1201 participants; MD $-5.68 \mathrm{mmHg}$; $95 \% \mathrm{CI}-9.68$ to -1.67 ; $\mathrm{I}^{2}=$ 60\%; DBP: 7 studies, 1201 participants; MD - 2.64 $\mathrm{mmHg}, 95 \% \mathrm{CI}-3.78$ to $-1.50 ; \mathrm{I}^{2}=0 \%$ ), and a lower
CRP level (3 studies, 123 participants; SMD -2.8; 95\% CI -2.90 to $-2.70, \mathrm{I}^{2}=0 \%$ ) than that of the usual care. However, there were no statistical differences in HbA1c or total cholesterol (TC) levels. Likewise, for the behavioral risk factor outcomes, patients who had received exercise management had longer distances on the 6-min walk (3 studies, 277 participants; SMD 0.70; 95\% CI 0.45

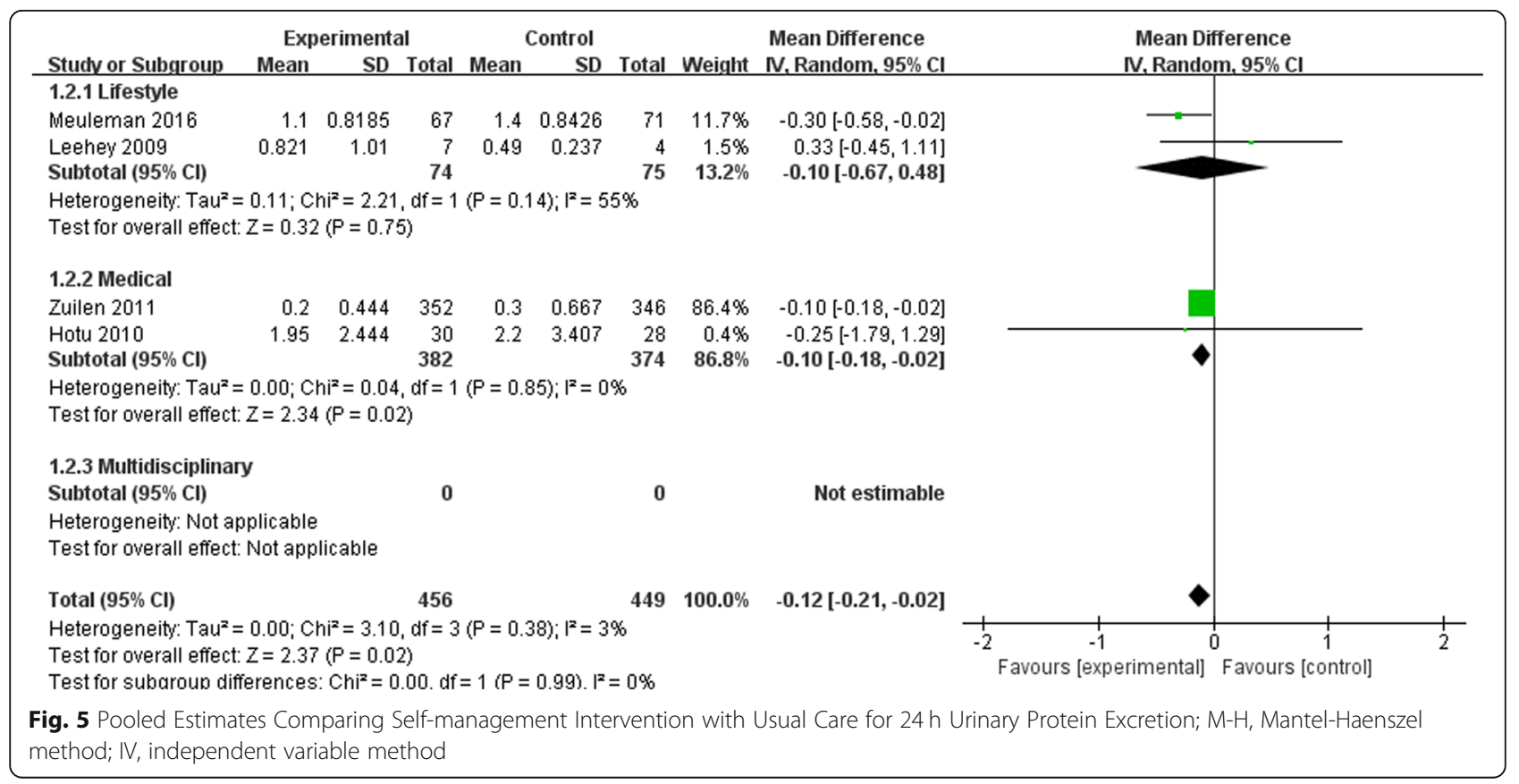


to $0.94 ; \mathrm{I}^{2}=0 \%$ ) than the control group. Regarding body weight and BMI, diet management appeared no better than usual care alone.

In the stratified analysis, it was observed that the multi-factorial modification group was associated with a significant decrease in HbA1c (3 studies, 344 participants; MD -0.68\%; 95\% CI -0.99,-0.36; $\mathrm{I}^{2}=65 \%$ ) compared with the usual care. (Additional file 1: Table S2).

\section{Adverse events of self-management intervention}

Four studies recorded adverse events (AEs); there were no specific descriptions for definition of all AEs; and no AEs occurred during the follow-up periods.

\section{Meta-regression and subgroup analyses}

Table 2 lists the results of univariate meta-regression analyses for exploring the potential sources of between-study heterogeneity. Though this review did not find evidence of specific contributors to heterogeneity, these effects might have differed according to baseline CKD stage, race, or the prevalence of disease.

\section{Discussion}

Although RRT has been available for decades in wealthy countries, most people with kidney failure have insufficient access to life-saving dialysis and kidney transplants. CKD care is an effective alternative, yet it has limitations: underutilization of health professionals in the care of patients with CKD, limited capacity for CKD surveillance, a general absence of national strategies to support CKD care, and poor integration of CKD care programs into national NCD control schemes [19]. A global change in the approach to CKD, from treatment to prevention, is imperative, especially in low- and middleincome countries that lack resources for $\mathrm{RRT}^{2}$.

\section{Results in relation to other studies and reviews}

To date, 6 systematic reviews have studied CKD management in general. While Lopez-Vargas 2016 [20], and Galbraith 2018 [21] provided education-based interventions, they did not focus on changing participants' beliefs. Although interventions might have been necessary for education, they are often insufficient, on their own, to produce behavioral change [22]. LEE (2016) [23] and Lin (2017) [24] identified CKD as including ESRD patients. In their study, dialysis participants' self-management intervention showed significant effects on self-efficacy, depression and health-related quality of life, while the effectiveness of self-management of non-dialysis CKD patients is limited. Helou (2016) [25] focused on multi-factorial management of diabetic kidney disease (DKD) patients. A recent systematic review has identified only 5 studies, and they are of varying methodological quality. This has led the authors to conclude "the effect of self-management programs in CKD (Stages 1-4) cannot be conclusively ascertained [26]."

Cardiovascular disease events, proteinuria and diabetes are associated with CKD progression, and the former have been the major cause of death in those with CKD. In our study, the results showed a significant drop in blood pressure and a lower $24 \mathrm{~h}$ urinary protein excretion among the self-management group. Long-term blood pressure drops reduce proteinuria and other indicators of structural damage. Early change in proteinuria may lead to an increased risk of ESRD and early death, and may also be associated with slower progression of kidney disease [27]. In the stratified analysis, it was observed that the multi-factorial modification group was associated with a significant decrease in HbA1c. Compared with CKD patients, co-existing CKD and diabetes patients carry a poor prognosis with increased all-cause mortality and cardiovascular mortality [28].

We also found that self-management intervention led to additional kidney protection, due to lower CRP levels and better exercise capacity. Evidence shows that exercise training results in improved physical performance and functioning among patients with CKD [29]. Also, regular participation in moderate-intensity exercise may enhance certain aspects of immune function and exert anti-inflammatory effects [30, 31]. Inflammatory markers such as CRP and anti-inflammatory cytokines correlate with underlying causes and consequences of the inflamed uremic phenotype such as oxidative stress, endothelial dysfunction, CVD, infections and protein-energy malnutrition (PEM, also referred to as PEW) [32]. As inflammatory biomarkers are sensitive predictors of outcomes in patients with ESRD, inflammation appears to be a target for preventive and therapeutic interventions in patients with CKD [33, 34]. This is consistent with our findings.

\section{Self-management theoretical frameworks}

Patient-oriented self-management is the cornerstone of chronic disease management, and optimized self-management is fundamental to controlling risk factors and improving disease management. Seeking to facilitate behavioral change rather than providing a purely educational program [35], self-management requires patients to shift away from passive education, and to become responsible for their own illness [36]. Patients are no longer a passive recipient of education; they are an active determiner of their health. Self-management intervention is a vehicle for helping patients develop skills and techniques to enhance self-care of their chronic conditions. Changing patients' beliefs is usually measured by asking "how confident are you" or "how sure are you" that under specific conditions they can achieve certain behaviors or physiological states. 


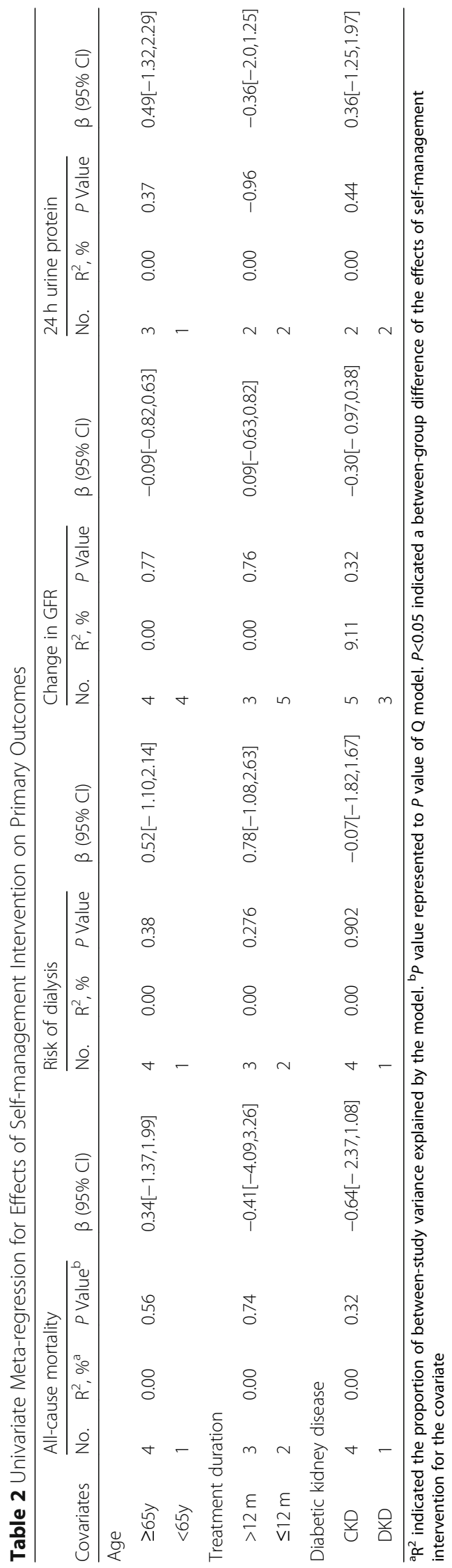


Our study consisted of the following 6 theoretical frameworks, with each model having unique aspects. These ranged from uni-dimensional variables to complex multi-dimensional constructs. Noar [37] analyzed the components of health behavior frameworks in terms of structures appertaining to attitudinal beliefs; self-efficacy and behavioral control beliefs; normative beliefs; risk-related beliefs and emotional responses; and intention, commitment and planning. We have updated the table after Noar (see Additional file 1: Table S3) showing how the structure and content of the models can be understood on multiple levels. The aforementioned theoretical frameworks for chronic diseases help refine the theoretical basis of intervention evaluations. However, at present, none are commonly used outside of research settings. Furthermore, the effectiveness of self-management for early-stage CKD is limited, and requires additional large-sample RCTs to assess the effectiveness of self-management intervention [38].

Additionally, computer-based machine learning algorithms can identify intervention at a practice level in real time to allow more focused and immediate correction of bias in NCD management [39]. For example, several studies have adopted novel machine learning algorithms to perform knowledge discovery on management of AEs or cancer complications [40, 41]. Therefore, the machine learning approach provides a general way to discover self-management strategies for NCDs [42].

\section{Strengths of this study}

This study has several strengths. First, the concept of self-management is debatable. Many prior studies have failed to distinguish it from health education or chronic disease management. Additionally, there are a variety of risk factors for CKD. Our study grouped similar risk factors together, and found that the effect of comprehensive intervention (lifestyle combined with medical behavior) is more effective. We believe this will be a new trend in future self-management intervention.

Second, current reporting of intervention content in published research articles and protocols lacks consistent terminology, making replicability difficult and uncommon. We concluded that there are 6 types of self-management frameworks, and this can provide reference for future self-management decision making.

\section{Limitations}

Our study also has several limitations. First, the effects of interventions on lifestyle and risk factor modification may require years for their results to modify surrogate and hard outcomes. A methodological limitation of the studies was the short-term follow-up might not distinguish kidney outcome differences, with most of the included studies had a follow-up time shorter than 2 years.
Second, there was heterogeneity in patient characteristics, trial designs and risk factor targets (obesity, hypertensive, salt intake, etc.) among the included studies. The number of included studies also limited the power for further exploration with multi-variate meta-regression or multi-level subgroup comparisons [43]. Therefore, we could only partially explain the influences of blood glucose on intervention effects. Third, funnel plots and Egger's test did not suggest publication bias, owing to the included studies (published studies only); such bias could still exist. Fourth, for the self-management framework, there is extensive heterogeneity in the body of research available, and it is uncertain what theory is best to predict (and ultimately to change) health behavior. Therefore, more integrative approaches are needed. Finally, only Chinese and English language reports were included. Consequently, we may be missing data from important studies published in other languages.

The drawback of a manual literature review is the time-consuming step of screening articles to select those that fulfill the requirements. Accordingly, some studies have leveraged computer-based topic analysis approaches to support literature review $[44,45]$. In the future, these approaches could be leveraged to facilitate the efficiency and effectiveness of systematic review [46].

\section{Conclusion}

We observed that self-management intervention provided additional benefits for neither renal outcomes nor all-cause mortality, when compared with standard treatments during a follow-up of 13.44 months in patients with CKD non-dialysis. However, it does show benefits for urine protein decline, blood pressure level, exercise capacity and CRP level. Hence, self-management intervention was beneficial for changing modifiable risk factors (e.g. proteinuria, blood pressure level, blood glucose level, exercise capacity) for the progression of kidney disease. It may have been beneficial in optimizing CKD patient outcomes and avoiding progression to ESRD, and thus may have improved survival.

Integration and ensuring the sustainability of healthcare self-management plans requires a large sample of RCT research and a unified and precise self-management intervention framework. These resources will help determine the ideal implementation for interventions.

\section{Additional file}

\footnotetext{
Additional file 1: Figure S1. Summary for Risk of Bias of Included Studies. Figure S2. Risk of Bias Graph of Included Studies. Figure S3. Funnel Plots, Contour-enhanced Funnel Plots, and Egger/Harbord Regression. Table S1. Association between Self-management Intervention and Standard Care by Subgroups. Table S2. Effects of Self-management on CKD Risk Factors. Table S3. Structure and Content of Self-management. (DOC 268 kb)
} 


\section{Abbreviations}

AEs: Adverts events; CEP: Certified exercise physiologists; Cl: Confidence interval; CKD: Chronic kidney disease; CRP: C-reactive Protein; DKD: Diabetic kidney disease; eGFR: Estimated Glomerular Filtration Rate; ESRD: End-Stage Renal Disease; KDIGO: Kidney Disease: Improving Global Outcomes; MD: Mean difference; MeSH: Medical Subject Headings; PEW: Protein-energy malnutrition; PRISMA: Preferred Reporting Items for Systematic Reviews and Meta-Analyses; RCT: Randomized controlled trial; RR: Relative risk; RRT: Renal replacement therapy; SMD: Standard mean difference; T1DM: Type 1 Diabetes; T2DM: Type 2 Diabetes; TC: Total cholesterol

\section{Acknowledgements}

We would like to thank the experts in this area who recommended studies for potential inclusion in this review.

\section{Funding}

This study was supported by Traditional Chinese Medicine Bureau of Guangdong Province, China (Chinese medicine preparation R\&D project: Sanqi oral preparation): XH Liu (2015KT1535); Guangzhou Science and Technology Program key projects: YF.W (2016201604030022); Guangdong Science and Technology Department: YF.W(2014A020221087) and Guangdong Provincial Hospital of Chinese Medicine Program: YF.W (YN2018ZWB04). These organizations had no role in study design, data collection, analysis, or interpretation of data. They also had no role in composing the manuscript.

\section{Availability of data and materials}

All datasets analyzed in this systematic review are referenced in the manuscript and Additional files.

\section{Authors' contributions}

XSL and YFW conceived and designed the experiments. SYP was the principal researcher and participated in all steps of the study (project development, data collection, database revision, data analysis, and drafting and revision of the article). JWH and JSH participated in developing the project, data analysis and article revision. LWL participated in database and manuscript revision. JHZ and SZ helped compose and revise the manuscript. LZ coordinated and conceived of the study and participated in project design, data collection supervision, data analysis, and drafting and revising the manuscript. All authors read and approved the final version of the article.

\section{Competing interest}

The authors declare that they have no competing interests.

\section{Ethics approval and consent to participate}

Not applicable.

\section{Consent for publication}

Not applicable.

\section{Publisher's Note}

Springer Nature remains neutral with regard to jurisdictional claims in published maps and institutional affiliations.

\section{Author details}

"The Second Medical College of Guangzhou University of Chinese Medicine, Guangzhou, China. ${ }^{2}$ Health Science Research, Mayo Clinic, Rochester, MN, USA. ${ }^{3}$ Renal Division, Peking University First Hospital, Beijing, China. ${ }^{4}$ Nephrology Department, Guangdong Provincial Hospital of Chinese Medicine, The Second Affiliated Hospital of Guangzhou University of Chinese Medicine, Guangzhou, China. ${ }^{5}$ Chronic Disease Management Department, Guangdong Provincial Hospital of Chinese Medicine, The Second Affiliated Hospital of Guangzhou University of Chinese Medicine, No. 111, Dade Rd, Yuexiu District, Guangzhou, Guangdong Province, China. ${ }^{6}$ EBM \& Clinical Research Service Group, Guangdong Provincial Hospital of Chinese Medicine, The Second Affiliated Hospital of Guangzhou University of Chinese Medicine, Guangzhou, China. ${ }^{7}$ Guangdong Provincial Academy of Chinese Medical Sciences, Guangzhou, China.
Received: 20 September 2018 Accepted: 21 March 2019

Published online: 26 April 2019

\section{References}

1. Go AS, Chertow GM, Fan D, McCulloch CE, C-y H. Chronic kidney disease and the risks of death, cardiovascular events, and hospitalization. $N$ Engl J Med. 2004;351(13):1296-305.

2. Webster AC, Nagler EV, Morton RL, Masson P. Chronic kidney disease. Lancet. 2017:389(10075):1238-52.

3. Keith DS, Nichols GA, Gullion CM, Brown JB, Smith DH. Longitudinal followup and outcomes among a population with chronic kidney disease in a large managed care organization. Arch Intern Med. 2004;164(6):659-63.

4. Di Angelantonio E, Danesh J, Eiriksdottir G, Gudnason V. Renal function and risk of coronary heart disease in general populations: new prospective study and systematic review. PLoS Med. 2007:4(9):e270.

5. Levin A, et al. Global kidney health 2017 and beyond: a roadmap for closing gaps in care, research, and policy. Lancet. 2017;390(10105):1888-917.

6. Peng S, Fan Y, Wang L, Wen A, Liu X, Liu H, Shen F. Leveraging Association Rule Mining to Detect Pathophysiological Mechanisms of Chronic Kidney Disease Complicated by Metabolic Syndrome. In: 2018 IEEE International Conference on Bioinformatics and Biomedicine (BIBM): 2018: IEEE; 2018. p. 1302-9. https://ieeexplore.ieee.org/abstract/document/8621226.

7. Institute of Medicine Committee on Identifying Priority Areas for Quality I. In: Adams K, Corrigan JM, editors. Priority Areas for National Action: Transforming Health Care Quality. Washington (DC): National Academies Press (US) Copyright 2003 by the National Academy of Sciences. All rights reserved; 2003.

8. McAndrew L, Schneider SH, Burns E, Leventhal H. Does patient blood glucose monitoring improve diabetes control? A systematic review of the literature. Diabetes Educ. 2007;33(6):991-1011 discussion 1012-1013.

9. Pillay J, Armstrong MJ, Butalia S, Donovan LE, Sigal RJ, Vandermeer B, Chordiya P, Dhakal S, Hartling L, Nuspl M, et al. Behavioral programs for type 2 diabetes mellitus: a systematic review and network meta-analysis. Ann Intern Med. 2015;163(11):848-60.

10. McManus RJ, Mant J, Haque MS, Bray EP, Bryan S, Greenfield SM, Jones MI, Jowett S, Little P, Penaloza C, et al. Effect of self-monitoring and medication self-titration on systolic blood pressure in hypertensive patients at high risk of cardiovascular disease: the TASMIN-SR randomized clinical trial. Jama. 2014;312(8):799-808.

11. Self-management interventions for chronic kidney disease: a systematic review. [http://www.crd.york.ac.uk/PROSPERO/display_record.php?ID= CRD42017059870]

12. Moher D, Shamseer L, Clarke M, Ghersi D, Liberati A, Petticrew M, Shekelle $P$, Stewart LA. Preferred reporting items for systematic review and metaanalysis protocols (PRISMA-P) 2015 statement. Syst Rev. 2015;4:1.

13. Clinical Practice Guideline for the Evaluation and Management of Chronic Kidney Disease [https://kdigo.org/wp-content/uploads/2017/02/KDIGO_ 2012_CKD_GL.pdf].

14. Higgins JPT, Altman DG, Gøtzsche PC, Jüni P, Moher D, Oxman AD, Savović J, Schulz KF, Weeks L, Sterne JAC. The Cochrane Collaboration's tool for assessing risk of bias in randomised trials. BMJ (Clinical research ed). 2011;343:d5928.

15. Borenstein $M$, Hedges LV, Higgins JP, Rothstein HR. A basic introduction to fixed-effect and random-effects models for meta-analysis. Res Synth Methods. 2010;1(2):97-111.

16. Egger M, Davey Smith G, Schneider M, Minder C. Bias in meta-analysis detected by a simple, graphical test. BMJ (Clinical research ed). 1997; 315(7109):629-34

17. Peters JL, Sutton AJ, Jones DR, Abrams KR, Rushton L. Contour-enhanced meta-analysis funnel plots help distinguish publication bias from other causes of asymmetry. J Clin Epidemiol. 2008;61(10):991-6.

18. Harbord RM, Egger M, Sterne JA. A modified test for small-study effects in meta-analyses of controlled trials with binary endpoints. Stat Med. 2006; 25(20):3443-57.

19. Bello AK, Levin A, Tonelli M, et al. Assessment of global kidney health care status. JAMA. 2017;317(18):1864-81.

20. Lopez-Vargas PA, Tong A, Howell M, Craig JC. Educational interventions for patients with CKD: a systematic review. Am J Kidney Dis. 2016;68(3):353-70.

21. Galbraith L, Jacobs C, Hemmelgarn BR, Donald M, Manns BJ, Jun M. Chronic disease management interventions for people with chronic kidney disease in primary care: a systematic review and meta-analysis. Nephrol Dial Transplant. 2018;33(1):112-21. 
22. Gibson PG, Powell H, Coughlan J, Wilson AJ, Hensley MJ, Abramson M, Bauman A, Walters EH. Limited (information only) patient education programs for adults with asthma. Cochrane Database Syst Rev. 2002;(2):Cd001005.

23. Lee MC, Wu SV, Hsieh NC, Tsai JM. Self-management programs on eGFR, depression, and quality of life among patients with chronic kidney disease: a meta-analysis. Asian Nurs Res. 2016;10(4):255-62.

24. Lin MY, Liu MF, Hsu LF, Tsai PS. Effects of self-management on chronic kidney disease: a meta-analysis. Int J Nurs Stud. 2017;74:128-37.

25. Helou N, Dwyer A, Shaha M, Zanchi A. Multidisciplinary management of diabetic kidney disease: a systematic review and meta-analysis. JBI Database System Rev Implement Rep. 2016;14(7):169-207.

26. Bonner A, Havas K, Douglas C, Thepha T, Bennett P, Clark R. Selfmanagement programmes in stages 1-4 chronic kidney disease: a literature review. J Ren Care. 2014;40(3):194-204.

27. Inker LA, Levey AS, Pandya K, Stoycheff N, Okparavero A, Greene T. Early change in proteinuria as a surrogate end point for kidney disease progression: an individual patient meta-analysis. Am J Kidney Dis. 2014;64(1):74-85.

28. Middleton RJ, Foley RN, Hegarty J, Cheung CM, McElduff P, Gibson JM, Kalra PA, O'Donoghue DJ, New JP. The unrecognized prevalence of chronic kidney disease in diabetes. Nephrol Dial Transplant. 2006;21(1):88-92.

29. Padilla J, Krasnoff J, Da Silva M, Hsu CY, Frassetto L, Johansen KL, Painter P. Physical functioning in patients with chronic kidney disease. J Nephrol. 2008;21(4):550-9.

30. Petersen AM, Pedersen BK. The anti-inflammatory effect of exercise. J Appl Physiol (Bethesda, Md : 1985). 2005;98(4):1154-62.

31. Beavers KM, Brinkley TE, Nicklas BJ. Effect of exercise training on chronic inflammation. Clin Chim Acta. 2010;411(11-12):785-93.

32. Carrero JJ, Yilmaz MI, Lindholm B, Stenvinkel P. Cytokine dysregulation in chronic kidney disease: how can we treat it? Blood Purif. 2008;26(3):291-9.

33. Meuwese CL, Stenvinkel P, Dekker FW, Carrero JJ. Monitoring of inflammation in patients on dialysis: forewarned is forearmed. Nat Rev Nephrol. 2011;7(3):166.

34. Meuwese CL, Snaedal S, Halbesma N, Stenvinkel P, Dekker FW, Qureshi AR, Barany P, Heimburger O, Lindholm B, Krediet RT. Trimestral variations of Creactive protein, interleukin- 6 and tumour necrosis factor-a are similarly associated with survival in haemodialysis patients. Nephrol Dial Transplant. 2010;26(4):1313-8.

35. Jones F, Riazi A. Self-efficacy and self-management after stroke: a systematic review. Disabil Rehabil. 2011;33(10):797-810.

36. Lorig KR, Holman H. Self-management education: history, definition, outcomes, and mechanisms. Ann Behav Med. 2003;26(1):1-7.

37. Noar SM, Zimmerman RS. Health behavior theory and cumulative knowledge regarding health behaviors: are we moving in the right direction? Health Educ Res. 2005;20(3):275-90.

38. Corbin JM. Unending work and care: managing chronic illness at home (JOSSEY BASS SOCIAL AND BEHAVIORAL SCIENCE SERIES): Jossey-Bass; 1988.

39. Shen F, Lee $Y$. Knowledge discovery from biomedical ontologies in cross domains. PLoS One. 2016;11(8):-e0160005.

40. Zhu Q, Tao C, Shen F, Chute CG. Exploring the pharmacogenomics knowledge base (PharmGKB) for repositioning breast cancer drugs by leveraging web ontology language (OWL) and cheminformatics approaches. Pac Symp Biocomput. 2014:172-82.

41. Feichen S, Hongfang L, Sohn S, Larson DW, Yugyung L. BmQGen: Biomedical query generator for knowledge discovery. In: 2015 IEEE International Conference on Bioinformatics and Biomedicine (BIBM): 9-12 Nov. 2015 2015; 2015. p. 1092-7. https://ieeexplore.ieee.org/document/7359833.

42. Shen F, Liu H, Sohn S, Larson DW, Lee Y. Predicate oriented pattern analysis for biomedical knowledge discovery. Intell Inf Manag. 2016;8(3):66-85.

43. Mayo-Wilson E. Reporting implementation in randomized trials: proposed additions to the consolidated standards of reporting trials statement. Am J Public Health. 2007;97(4):630-3.

44. Li D, Wang Z, Wang L, Sohn S, Shen F, Murad MH, Liu H. A text-mining framework for supporting systematic reviews. Am J Inf Manage. 2016;1(1):1.

45. Li D, Wang Z, Shen F, Murad MH, Liu H. Towards a multi-level framework for supporting systematic review - A pilot study. In: Bioinformatics and Biomedicine (BIBM), 2014 IEEE International Conference on: 2014: IEEE; 2014. p. 43-50. https:/ ieeexplore.ieee.org/document/6999266

46. Zhang Y, Li D, Tao C, Shen F, Liu H. An integrative computational approach to identify disease-specific networks from PubMed literature information. In: 2013 IEEE International Conference on Bioinformatics and Biomedicine (BIBM): 2013: IEEE; 2013. p. 72-5. https://ieeexplore.ieee.org/document/6732738
47. Meuleman Y, Hoekstra T, Dekker FW, Navis G, Vogt L, van der Boog PJM, Bos WJW, van Montfrans GA, van Dijk S. Sodium restriction in patients with CKD: a randomized controlled trial of self-management support. Am J Kidney Dis. 2017:69(5):576-86.

48. Rossi AP, Burris DD, Lucas FL, Crocker GA, Wasserman JC. Effects of a renal rehabilitation exercise program in patients with CKD: a randomized, controlled trial. Clin J Am Soc Nephrol. 2014;9(12):2052-8.

49. Teng HL, Yen M, Fetzer S, Sung JM, Hung SY. Effects of targeted interventions on lifestyle modifications of chronic kidney disease patients: randomized controlled trial. West J Nurs Res. 2013;35(9):1107-27.

50. Mustata S, Groeneveld S, Davidson W, Ford G, Kiland K, Manns B. Effects of exercise training on physical impairment, arterial stiffness and health-related quality of life in patients with chronic kidney disease: a pilot study. Int Urol Nephrol. 2011;43(4):1133-41.

51. Campbell KL, Ash S, Bauer JD. The impact of nutrition intervention on quality of life in pre-dialysis chronic kidney disease patients. Clin Nutr. 2008; 27(4):537-44.

52. Flesher M, Woo P, Chiu A, Charlebois A, Warburton DE, Leslie B. Selfmanagement and biomedical outcomes of a cooking, and exercise program for patients with chronic kidney disease. J Ren Nutr. 2011;21(2):188-95.

53. Leehey DJ, Moinuddin I, Bast JP, Qureshi S, Jelinek CS, Cooper C, Edwards LC, Smith BM, Collins EG. Aerobic exercise in obese diabetic patients with chronic kidney disease: a randomized and controlled pilot study. Cardiovasc Diabetol. 2009;8:62.

54. Mekki K, Bouzidi-bekada N, Kaddous A, Bouchenak M. Mediterranean diet improves dyslipidemia and biomarkers in chronic renal failure patients. Food Funct. 2010;1(1):110-5.

55. Howden EJ, Coombes JS, Strand H, Douglas B, Campbell KL, Isbel NM. Exercise training in CKD: efficacy, adherence, and safety. Am J Kidney Dis. 2015;65(4):583-91.

56. Byrne J, Khunti K, Stone M, Faroogi A, Carr S. Feasibility of a structured group education session to improve self-management of blood pressure in people with chronic kidney disease: an open randomised pilot trial. BMJ Open. 2011;1(2):e000381.

57. van Zuilen $A D$, Blankestijn PJ, van Buren $M$, ten Dam MA, Kaasjager KA, Ligtenberg G, Sijpkens YW, Sluiter HE, van de Ven PJ, Vervoort G, et al. Nurse practitioners improve quality of care in chronic kidney disease: two-year results of a randomised study. Neth J Med. 2011; 69(11):517-26.

58. Hotu C, Bagg W, Collins J, Harwood L, Whalley G, Doughty R, Gamble G, Braatvedt G. A community-based model of care improves blood pressure control and delays progression of proteinuria, left ventricular hypertrophy and diastolic dysfunction in Maori and Pacific patients with type 2 diabetes and chronic kidney disease: a randomized controlled trial. Nephrol Dial Transplant. 2010;25(10):3260-6.

59. Williams A, Manias E, Liew D, Gock H, Gorelik A: Working with CALD groups: Testing the feasibility of an intervention to improve medication selfmanagement in people with kidney disease, diabetes, and cardiovascular disease, vol. 8; 2012.

60. Joboshi H, Oka M. Effectiveness of an educational intervention (the encourage autonomous self-enrichment program) in patients with chronic kidney disease: a randomized controlled trial. Int J Nurs Stud. 2017;67:51-8.

61. Ishani A, Christopher J, Palmer D, Otterness S, Clothier B, Nugent S, Nelson $D$, Rosenberg ME. Telehealth by an Interprofessional team in patients with CKD: a randomized controlled trial. Am J Kidney Dis. 2016;68(1):41-9.

62. Steed L, Lankester J, Barnard M, Earle K, Hurel S, Newman S. Evaluation of the $U C L$ diabetes self-management programme (UCL-DSMP): a randomized controlled trial. J Health Psychol. 2005;10(2):261-76.

63. Williams A, Manias E, Walker R, Gorelik A. A multifactorial intervention to improve blood pressure control in co-existing diabetes and kidney disease: a feasibility randomized controlled trial. J Adv Nurs. 2012;68(11):2515-25.

64. Chan JC, So WY, Yeung CY, Ko GT, Lau IT, Tsang MW, Lau KP, Siu SC, Li JK, Yeung VT, et al. Effects of structured versus usual care on renal endpoint in type 2 diabetes: the SURE study: a randomized multicenter translational study. Diabetes Care. 2009:32(6):977-82

65. Chen SH, Tsai YF, Sun CY, Wu IW, Lee CC, Wu MS. The impact of selfmanagement support on the progression of chronic kidney disease--a prospective randomized controlled trial. Nephrol Dial Transplant. 2011; 26(11):3560-6. 